P\&A Año 3, N. ${ }^{\circ} 5$

julio-diciembre 2018

pp. [65]-77

\title{
Resumen
}

Este ensayo reconoce el rol del esquisse en la enseñanza de arquitectura en los primeros talleres de diseño de Lima como un ejercicio proyectual de corta duración. Si bien su uso es constantemente planteado como una primera aproximación al estudiante iniciado, es escasa la reflexión surgida a partir la variedad de tipologías que puede presentar. Es así que, a partir del análisis de los casos de estudio, se destaca los aspectos esenciales en arquitectura que cada esquisse aborda, así como los formatos y usos más propuestos en su elaboración para articular los contenidos del taller de diseño. Finalmente, se confirma su capacidad de perfilar un taller inicial.

Palabras clave: Esquisse, enseñanza en arquitectura, taller de diseño

\section{El esquisse en la enseñanza de arquitectura en los primeros talleres de diseño de Lima*}

\section{The esquisse in the teaching of architecture in the first design workshops in Lima}

\section{Est. Arq. Gianfranco Ricardo Cueto Narváez**}

Recibido: 20 de marzo de 2018

Aceptado: 26 de junio de 2018

\begin{abstract}
This essay surveys the esquisse's role im the architecture teaching in the first design studio of three faculties of Lima city as a short length exercise. Even though its use is constantly proposed as a first approach to the student, the reflection arised from the variety of typologies that it can present is limited. From the analisis of the case studies, the essential aspects in architecture that each esquisse addresses, as well as the most proposed formats and applications in its elaboracion in order to articulate the contents of the design studio, are highlighted. Finally, its capacity to evaluate an initial studio is confirmed.
\end{abstract}

Keywords: : Esquisse, architecture teaching, design workshop

* El artículo resulta de la compilación del material utilizado para la investigación realizada para el curso Taller de Investigación (2019-1) en la FAU-PUCP. Se declara no tener conflictos de intereses en la publicación de este artículo

**- Estudiante de arquitectura de la Facultad de Arquitectura y Urbanismo de la Pontificia Universidad Católica del Perú 


\section{Introducción}

Desde la formación academicista del arquitecto en la escuela de Bellas Artes de París hasta los cambios que ha sufrido su definición hasta la actualidad, el esquisse ha venido cumpliendo un rol importante en la formación del arquitecto como un ejercicio proyectual de corta duración. Si bien, actualmente su uso puede ser fácilmente adoptado en cursos que involucren un proceso proyectual, es en Taller de diseño cuya presencia es más abordada. Sin embargo, a pesar de su historia y su presencia en la actualidad, es escasa la producción teórica referida a esta.

Es así que el presente ensayo tiene la finalidad de abordar al esquisse como una de las herramientas pedagógicas que viene formando parte importante en las prácticas de enseñanza en arquitectura en el curso de Taller de diseño; y, especialmente, en los niveles iniciales. Para esto, se hizo un levantamiento de los esquisses realizados en tres talleres de diseño uno de tres facultades de arquitectura en Lima durante el ciclo académico 2016-2 y 2017-2.

Por tratarse de una propuesta que parte de los análisis individuales de cada esquisse, para, finalmente, perfilar la enseñanza de cada taller de diseño, se empleó una metodología inductiva. Para esto, se tomó en cuenta las características tipológicas de cada esquisse a partir de su observación; y, también, los aspectos esenciales en arquitectura que le corresponden.

\section{El esquisse, antecedentes y clasificacio- nes}

El esquisse (boceto) tiene su origen en la formación academicista en la Escuela de Bellas Artes de París; el cual, junto a los projes rendus (proyectos terminados), conformaban los concours (concursos) de diseño que debía realizar el alumno de forma obligatoria para poder ser evaluado. Si bien el project rendu consistía en la elaboración de un proyecto en toda su complejidad, el esquisse consistía en elaborar dibujos proyectuales donde se evidencien las intenciones compositivas del alumno en la elaboración de proyectos de mucha menor escala. Esta, a su vez, se desarrollaba bajo condiciones estrictas en un tiempo determinado de 9 a 12 horas (Chafee, 1977, pp. 19-20).

Sin embargo, en lo que respecta a su uso contemporáneo, el esquisse refiere a "ejercicios de corta duración (tanto individuales y grupales) que se establecen en intervalos dentro del pasaje de un largo proyecto de diseño o un semestre académico" (Beynon \& Tucker, 2012, p. 178). Es así que, en su propia elaboración, existe un constante proceso de producción, reflexión y aprendizaje durante el tiempo limitado estipulado. Además, es una herramienta didáctica que no solo es flexible en sus indicaciones y aspectos a evaluar, sino también por su cualidad de poder aislar un aspecto del proyecto, de forma abstracta, en un ejercicio puntual.

Si bien el esquisse está constantemente utilizado en la enseñanza en arquitectura, su uso varía dependiendo de la presencia (o ausencia) del proyecto arquitectónico como protagonista dentro del taller de diseño. Esto es porque, en talleres que comprenden la elaboración lineal de un proyecto arquitectónico, el esquisse tienen la función de concentrarse en alguna etapa y aspecto específico del proyecto, permitiendo al alumno concentrarse en su desarrollo en relativo aislamiento. Por otro lado, en talleres donde no se resuelve un proyecto arquitectónico (principalmente los primeros), el esquisse cobra un rol más protagónico e independiente, donde buscan generar una indagación sobre los conceptos esenciales de la disciplina arquitectónica (tectónico, espacial, antropométrico, etc.). Finalmente, la presente investigación se centra en esta última categoría, a partir del análisis de las tipologías reconocidas desde sus instrucciones, sus formatos y la función que cumplen.

\section{Las instrucciones}

Para plantear las instrucciones de cada esquisse, se "incorpora la variable lúdica para introducir al estudiante en una experiencia de interacción comunicativa; por lo tan- 
to, se establecen reglas, penalidades y retos que estructuran la base competitiva del “juego"” (Fabbri \& Tapia, 2015, p. 115). A su vez, estas deben estar claramente establecidas, $y$, sin embargo, deben poder fomentar una flexibilidad en cuanto a posibles respuestas para un mismo problema. En conclusión, si bien para motivos de la investigación, las instrucciones no representan una variable cualitativa para perfilar los esquisse, se debe evitar un elevado nivel de abstracción en el planteamiento de sus indicaciones, así como su exagerada acotación.

\section{Los formatos}

Los formatos, para el esquisse, corresponden a las herramientas de representación de las ideas proyectuales de cada alumno "para la aprehensión, la comprensión y la comunicación del diseño" (Bertero, 2012, p. 62). Por lo tanto, estos no solamente cumplen la función de traducir las indicaciones mediante un producto final, sino que son un proceso de exploración y aprendizaje por sí mismos. Por lo tanto, el resultado permite, finalmente, la correcta legibilidad del proceso de diseño efectuado, así como un resultado comunicable de las ideas entre el alumno y la cátedra. En conclusión, los formatos de representación están intrínsecamente relacionados en la enseñanza de arquitectura.

En la formación en arquitectura en los primeros talleres de diseño, se pueden reconocer formatos típicos como lo son los croquis tridimensionales (maquetas y modelos) y el dibujo proyectual (bocetos, cortes, plantas, elevaciones, etc.). Sin embargo, a partir del análisis de los estudios de caso, se han podido identificar nuevas propuestas como los formatos digitales cuya elaboración correspondería más comúnmente a los talleres de nivel superior. A su vez, se reconoce un nuevo formato que identificamos como formato corporal, el cual involucra el uso del cuerpo como parte de las dinámicas.

\section{Las funciones}

En los primeros talleres de diseño, la función del esquisse no depende de su articulación con un proyecto de arquitectura elaborado a lo largo del semestre académico. Por el contrario, al no plantearse un proyeto arquitectónico, el esquisse se caracteriza por su flexibilidad al momento de articularse con el resto de dinámicas que compone el taller de diseño. Finalmente, las categorías de esquisse son reconocidas a partir su función individual reconocida a partir del análisis de los casos de estudio.

Por lo tanto, se identifica en primer lugar, al esquisse independiente, pues no guarda relación de continuidad con las dinámicas posteriores, así como no se articula con el esquisse inmediatamente anterior.

En segundo lugar, se encuentra el preesquisse, el cual tiene la función de ser un punto de partida para un esquisse posterior de mayor complejidad. Tienen un nivel de complejidad y dificultad inicial con respecto al siguiente esquisse, pero comparten el mismo enfoque $\mathrm{y}$ formato.

La tercera categoría corresponde a los esquisse continuos que se caracteriza por tener un mismo o mayor nivel de complejidad que el ejercicio anterior, sin embargo, considerando en gran medida los enfoques y formatos abarcados. Por lo tanto, estos tienden a pertenecer a una misma unidad temática dentro del taller.

La cuarta y última categoría es el esquisse síntesis: Se caracteriza principalmente por ser una sumatoria de los enfoques realizados a lo largo de las dinámicas anteriores. Por lo tanto, tienen el mayor nivel de complejidad entre los ejercicios, así como el mayor tiempo de duración para su elaboración. Marcan el final de las unidades temáticas, así como del semestre académico.

\section{La enseñanza en arquitectura a partir de sus conceptos fundamentales}

La presente investigación adopta la clasificación propuesta por la arquitecta Cristina Dreifuss donde señala que "los talleres de diseño pueden agruparse en enfoques, 
es decir, en aquel o aquellos aspectos de la arquitectura a los que se dan mayor importancia" (Dreifuss, 2015, p. 78). Esta clasificación, como bien menciona, parte del reconocimiento de conceptos fundamentales en arquitectura, los cuales son expuestos en los talleres de diseño a partir de enfoques. Es así que finalmente, los conceptos fundamentales inicialmente reconocidos se emplean para la enseñanza en arquitectura en los primeros talleres de diseño a través de los esquisse.

\section{El enfoque Espacial}

Dentro de los primeros talleres de diseño, el enfoque espacial refiere a las cualidades visuales, de luminosidad, y de las dimensiones y escalas que derivan los límites del ambiente (Ching, 2006, p. 92). Si bien, dentro de un nivel inicial, ya se puede abordar límites formales como muros, losas, vanos; al tratarse de la primera aproximación al concepto de espacio, este se aborda a partir de su abstracción. Por lo tanto, si bien ya los límites formales arquitectónicos no son utilizados, se considera el espacio como espacio libre y vacío, pero delimitado.

\section{El enfoque técnico-tectónico}

La habilidad técnica en la arquitectura, a diferencia de otros campos artísticos, es esencialmente más intensa, obligándola indiscutiblemente a anticipar una metodología en su construcción. Sin embargo, esta cualidad técnica no puede ser vista como una "carga" o limitante al momento de desarrollar arquitectura, pues es mediante esta que se puede impulsar su desarrollo; potenciándola y dándole forma. Por lo tanto, el enfoque busca la noción del sistema constructivo a utilizarse, así como las propiedades del material a utilizar, considerando su estructura y el correcto lenguaje en su uso.

\section{El enfoque territorial}

El entendimiento del entorno cotidiano; ya sea natural, ciudad, etc., es fundamental para toda proyección arquitectónica, pues esta ocurre dentro de un territorio existente, no irreal ni abstracto. Para esto, Muñoz
(2008) identifica de forma explícita tres condiciones del territorio que desembocan en un número de variables tanto cualitativas como cuantitativas, de las cuales puede devenir el análisis del lugar. Estas consisten en sus características físicas, sus características perceptuales y sus características culturales.

\section{El enfoque de usuario-función}

Dentro del contexto de los primeros talleres de diseño, se puede "entender la arquitectura como albergue o escenario de las actividades humana" (Dreifuss, 2015, p. 84). Por lo tanto, el problema de la función radicaría principalmente en la definición de su propio término, donde puede referirse tanto a los programas (y su espacialidad) en respuesta a los usos establecidos de los usuarios; y a su vez, también puede referir a su función simbólica atribuible (Ramón, 2002, p. 112). Finalmente, las características cualitativas utilizadas para reconocer el enfoque funcional dependen del uso del usuario que albergará el espacio, así como los ejercicios realizados en torno al hábitat y cómo las características del usuario las moldean.

\section{El enfoque estético-formal}

En el contexto del primer taller de diseño, y en la enseñanza de arquitectura en general, existe la dificultad para "definir las razones cualitativas que determinan que un objeto 'se vea bien' o no" (Dreifuss, 2015, p. 83). Para esto, dentro de la investigación realizada por Dreifuss (2015), se menciona dos aproximaciones para determinar la buena o mala ejecución del alumno en torno al ejercicio. En primer lugar, las propuestas ligadas a la antropometría y la búsqueda de proporción a partir de esta. Y, en segundo lugar, las propuestas ligadas a la forma y su capacidad de deformación. Finalmente, ambas experiencias constituyen "una toma de conciencia de la experiencia estética, que busca acercarla al ámbito de lo racional y explicarla según ciertas normas o reglas" (Dreifuss, 2011, p. 66), donde el quehacer del alumno pvuede ser cuestionado y justificado, tanto por él como por el profesor 


\section{El enfoque antropométrico}

Desde la propuesta de Le Corbusier sobre El Modulor, publicado en 1942, se reconoce una intención de estandarización de las medidas en arquitectura para una mayor eficiencia no solo en su producción, sino también en sus costos. Esto se debe a que finalmente, la arquitectura "debe responder a sus necesidades no solo de uso, sino también de dimensión" (Dreifuss, 2015, p. 86). Por lo tanto, reconocer un enfoque antropométrico en los primeros talleres de diseño, se requiere la búsqueda de la noción del cuerpo humano en cuanto sus dimensiones y proporciones por parte del alumno.

\section{Los 3 talleres de diseño}

El taller de diseño se reconoce como el curso principal en la malla curricular de toda facultad de arquitectura de Lima y es el espacio donde se efectúa la enseñanza en arquitectura a partir de un aprender haciendo por medio de ejercicios o un proyecto. Por lo tanto, cumple un rol protagónico en la formación del futuro arquitecto, pues, además, "constituye la síntesis de todas las materias de la misma" ( $\mathrm{Mu}-$ ñoz, 2008, p. 195) vistas en el resto de cursos.

Para motivos de la investigación, se selecciona tres talleres de diseño uno (1) en tres diferentes facultades de arquitectura en Lima. Los criterios de selección dependieron de la antigüedad que tenía cada facultad, así como su relevancia en la historia. Por otra parte, el listado del portal Ponte en Carrera donde evidencia el promedio salarial de un egresado de cada facultad, factor que fue considerado para la selección de los casos de estudio. Por lo tanto, la Fau-PUCP; la Fau-URP y la Faua-UNI terminan encabezando estas condiciones. Finalmente, la selección del ciclo académico a estudiar dependió de la cantidad de información que se pudo compilar de cada ciclo.
Taller de diseño 1-Aldo Mantovani Busato FAU-PUCP (2016-2)

\section{Instrucciones}

Con respecto a las instrucciones, en el Taller 1 del arquitecto Aldo Mantovani, tienen un nivel de abstracción intermedio, debido a la realización de solamente 7 ejercicios durante el ciclo académico. Por lo tanto, existe un nivel de abstracción controlado que no solamente fomenta soluciones creativas para un mismo problema, sino también una adecuada motivación. Por otro lado, cabe señalar que a cada esquisse le corresponde dos formatos. Al principio, las indicaciones para las presentaciones finales iban dirigidas a la realización del formato principal (croquis tridimensional y corporal), las cuales conllevan un nivel de abstracción mayor en su realización. Sin embargo, acompañando a estas, también estaban las indicaciones para su crítica a partir del formato de dibujo (planos y paneles explicativos) a mano.

\section{Formatos}

Con respecto a los formatos-tipologías, existe un marcado trabajo con dos formatos: el croquis tridimensional y el dibujo (ver figura 1). Sin embargo, estos formatos no están distribuidos en esquisse diferentes; por el contrario, están presentes al mismo tiempo en cada ejercicio. Esta dualidad considerada en la presentación final de cada esquisse permite dos puntos de vista hacia un mismo ejercicio. Es así que, el alumno genera un aprendiza-
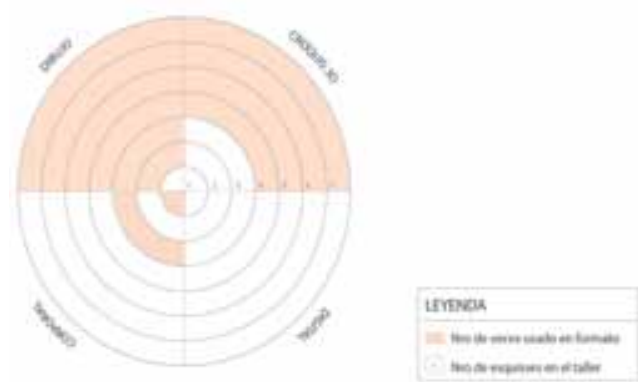

Figura 1. Formatos de los esquisse en el taller de diseño 1 de la FAU-PUCP (2016-2) 
je no solamente a partir de la exploración material, sino que además se pone a prueba a partir del formato de dibujo geométrico.

Por otra parte, es el primer taller que explora con los formatos corporales (vistos en los esquisse 1 y 3 ). Su escasa aparición en los talleres iniciales de diseño tiene que ver con su difícil flexibilidad para el uso en formatos diferentes al antropométrico. Sin embargo, por lo considerado en los estudios de casos, permite una mayor exploración sobre este enfoque, además del conocimiento de las dimensiones y proporciones. Esto se debe a que se reconoce al hombre como una estructura articulada, cuyo sistema de movimientos naturales está condicionado a esta.

\section{Funciones}

Los esquisse en el taller de diseño 1 de la FAU-PUCP (durante el ciclo 2016-2) consisten en una serie de 7 ejercicios puntuales. Posteriormente, se culmina en la elaboración de un esquisse síntesis que busca abarcar los aspectos vistos anteriormente $\mathrm{y}$ añadiendo la variable del espacio como confinador de actividades. Por lo tanto, los esquisse no tienen una función dentro de un proyecto mayor. Finalmente, el taller no busca una conclusión a partir de la elaboración de un proyecto arquitectónico. Por el contrario, cada ejercicio es independiente al proyecto, pues cada uno enseña sobre aspectos fundamentales de la disciplina de manera abstracta durante la elaboración de los 7 ejercicios.

Finalmente, y como se evalúa en los estudios de casos, las temáticas se desarrollan a lo largo del taller por medio de esquisse continuos, siendo el hombre la temática que estaría presente a lo largo de este desarrollo como generador final del espacio. Por lo tanto, el carácter continuo de cada esquisse representa la intención del taller en partir del enfoque antropométrico e ir sumando complejidad a partir de añadir nuevas unidades temáticas (territorio y espacio).

\section{Enfoques}

Sobre los enfoques abarcados en los esquisse, el taller busca trabajar de forma prota- gónica con los enfoques antropométrico, espacial y territorio (ver figura 2). Además, se reconocen los enfoques estético-formal y usuario-función de manera complementaria. Sin embargo, en ningún esquisse del taller se abarca los enfoques técnico-tectónico. Finalmente, el aspecto antropométrico se encarga de estructurar todas las dinámicas del taller, pues se partía del hombre para, posteriormente, engranar con los nuevos enfoques.

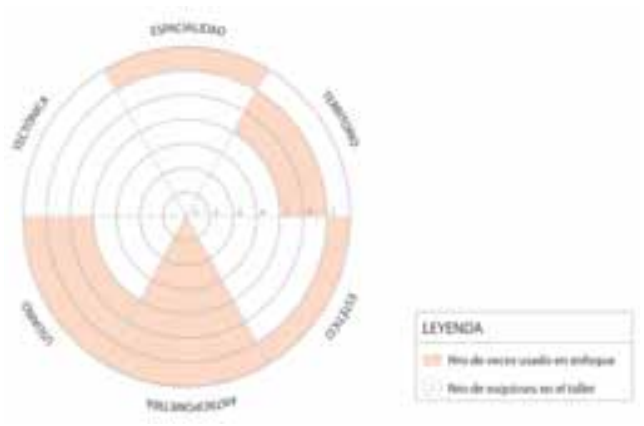

Figura 2. Enfoques de los esquisse en el taller de diseño 1 de la FAU-PUCP (2016-2)

Con respecto al aspecto antropométrico, no solamente funciona como punto de partida de las primeras dinámicas del taller, sino que su uso es constante a lo largo de este, abarcando los 7 esquisse en su totalidad. Esto se debe a que en todos se parte de la noción del cuerpo humano como sistema de noción de medidas. A su vez, se considera las proporciones del hombre con respecto al tamaño de su cabeza; de tal forma que el alumno debía expresar esta noción constantemente a partir del formato gráfico de dibujo proyectual.

Por otra parte, se reconoce que el taller inicia con una exploración exclusivamente antropométrica, tomando al hombre como unidad de medida durante los primeros esquisse. Posteriormente, en el esquisse tres y cuatro, se enfatiza en comprender de primera mano el cuerpo a partir de su estructura ósea y las articulaciones; esto se consigue mediante la posición en movimiento, la cual es posteriormente traducida en un croquis tridimensio- 
nal. Finalmente, los últimos esquisse corresponden al hombre y su desplazamiento para intervenir en el territorio y generar el espacio. Finalmente, esta distribución articulada y de ascendente complejidad permite una comprensión ordenada del taller, y la importancia del hombre en el proceso proyectual.

Durante los últimos tres (3) ejercicios, se reconoce el enfoque de usuario-función, el cual está estrictamente ligado a la antropometría. Esto se debe a que el hombre, en los últimos dos ejercicios, no solamente genera un desplazamiento; sino que este está condicionado a una actividad específica. Por lo tanto, la propuesta espacial en ambos esquisse estarán en función a la actividad a realizarse en estos. Sin embargo, esta cualidad se ve en mejor medida en el esquisse siete, pues se debe reconocer una relación directa entre la actividad propuesta, y el espacio que la contiene.

El enfoque territorial se abarca en ascendente complejidad durante los ejercicios cinco y seis. Esto a partir del reconocimiento de las características perceptuales y físicas de un terreno escogido. A pesar de ser realizado en, únicamente, dos ejercicios, este está bien resuelto en cuanto el alumno puede conocer de primera mano el espacio a intervenir. Por lo tanto, su exploración y justificación para la intervención será más profunda y sensible.

El enfoque espacial se toma en el taller como resultado generado a partir de la antropometría y su desplazamiento. Si bien se resuelven las condiciones de iluminación, límites formales, dimensión y escala, y la forma durante un solo ejercicio, este tiene una duración de cuatro semanas y media (nueve clases). Por lo tanto, ocupa casi el $40 \%$ del total de tiempo del taller.

Finalmente, además de abarcar de manera exitosa los enfoques, el potencial está en el uso de la antropometría como columna vertebral de estos temas. Por lo tanto, existe un nivel de complejidad ascendente, sin perder la noción del hombre como sujeto generador de arquitectura. Sin embargo, no todos los enfoques son acumulativos, y esto se evidencia en que el esquisse síntesis no abarca el aspecto territorial de forma explícita.

\section{Taller de diseño 1-Manuel Flores Caballero- FAUA-UNI (2016-2)}

\section{Instrucciones}

Con respecto a las instrucciones en el Taller uno (1) del profesor Manuel Flores, se establecen reglas y penalidades explícitas (bastante acotadas) en el desarrollo de cada ejercicio. Por otro lado, si bien no se diferencia indicador cualitativo para distinguir las características de estos, esto se debe a los muchos esquisse que se incluyen en el taller, y que, por lo tanto, cada ejercicio individual requerirá de una inmediata comprensión para su rápida elaboración. Por lo tanto, si bien estas instrucciones están claramente establecidas, se puede reconocer, en su planteamiento, una suficiente flexibilidad en cuanto posibles respuestas hacia el mismo problema. Esto se pone en evidencia al reconocer las diversas soluciones propuestas en clase por los mismos alumnos.

\section{Formatos}

Con respecto a los formatos-tipologías elaboradas durante el taller, existe una marcada preferencia por los croquis tridimensionales (ver figura 3). Si bien se plantea el uso intensivo del dibujo como herramienta de análisis de la realidad y del collage, es la representación tridimensional la herramienta de la expresión final de una idea. El uso de este formato inicia desde el planteamiento del segundo esquisse, principalmente para generar en el alumno una relación y exploración directa con el material, así como sus propiedades y naturaleza. Además, se siguió usando en el resto de ejercicios posteriores con otras funciones. Este proceso, finalmente, se diferencia de las representaciones bidimensionales exclusivas del dibujo (o la pantalla de una computadora) para estimular los sentidos a partir del punto de vista volumétrico, revelando así nuevas facultades a partir de esta. 

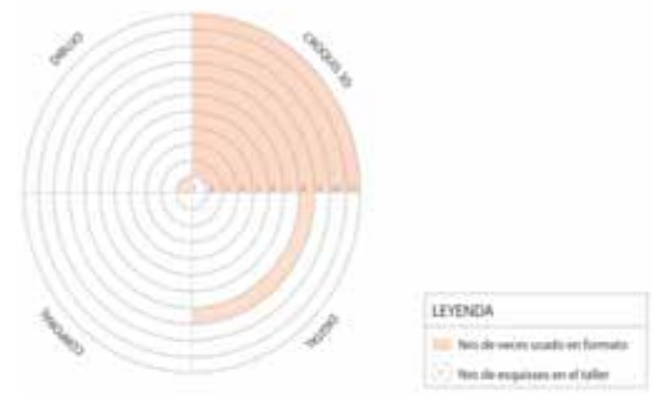

Figura 3. Formatos de los esquisse en el taller de diseño 1 de la FAUA-UNI (2016-2)

En conclusión, no se enfocaba en un aprendizaje que involucre formatos diferentes al croquis tridimensional, limitando el uso del formato de dibujo exclusivamente a las bitácoras. Por otro lado, los formatos digitales (salvo durante las exposiciones con ppt) y corporales no se presentan en ningún punto del ciclo. Además, lo más destacable es el uso del formato de croquis tridimensional para la comprensión tectónica de materiales de fácil manipulación, explorando desde sus propiedades estructurales, hasta la producción espacial a partir de estos.

\section{Funciones}

Sobre las funciones de los esquisse en el taller de diseño 1 de la FAUA-UNI (durante el ciclo 2016-2), estos culminan en la elaboración de un proyecto arquitectónico a modo de síntesis de las unidades temáticas vistas anteriormente. Sin embargo, esto no significa que cada esquisse responde a algún aspecto específico a este proyecto (anteproyecto, material, detalle constructivo, etc.). Por el contrario, cada esquisse es autónomo e independiente a este pues se enfocan en abarcar conceptos fundamentales de la disciplina arquitectónica a partir de su propia resolución. Por lo tanto, y a diferencia de los esquisse articulados dentro de un proyecto mayor, la abstracción en los planteamientos de estos conceptos fundamentales son lo esencial al momento de plantear cada dinámica. Finalmente, si bien culmina en la elaboración de un objeto ar- quitectónico, esto no resulta necesariamente en algo negativo o positivo; sino, en cómo se ha llegado a este punto a partir de las unidades temáticas, y qué ha abarcado cada una.

Finalmente, la primera unidad temática del taller (sin contar el esquisse 1) abarca el enfoque técnico-tectónico. Esta está conformada por los esquisse dos, tres y cuatro (2, 3 y 4) de carácter continuo. Posteriormente, la siguiente unidad temática introduce el enfoque espacial mediante un ejercicio práctico (esquisse 5) y uno de introducción teórica (esquisse 6), los cuales concluyen en un esquisse síntesis (esquisse 7). Luego, se involucra una tercera unidad temática correspondiente al enfoque territorial por medio de dos esquisse complementarios entre ellos (esquisse 8 y 9). Finalmente, tanto el esquisse 9 como el 10, consisten en la última unidad temática sobre los aspectos tectónicos y espaciales. Finalmente, el proyecto se compone de la suma de estas dos últimas unidades temáticas, sin embargo, el enfoque tectónico se pierde desde la exploración con la materia en el ejercicio diez (10). Por lo tanto, si bien la espacialidad parte de esta exploración del material (cartulina folcote), el proyecto no contempla un sistema constructivo que la condicione.

\section{Enfoques}

Sobre los enfoques abarcados en los esquis$s e$, el taller enfatiza de forma explícita los
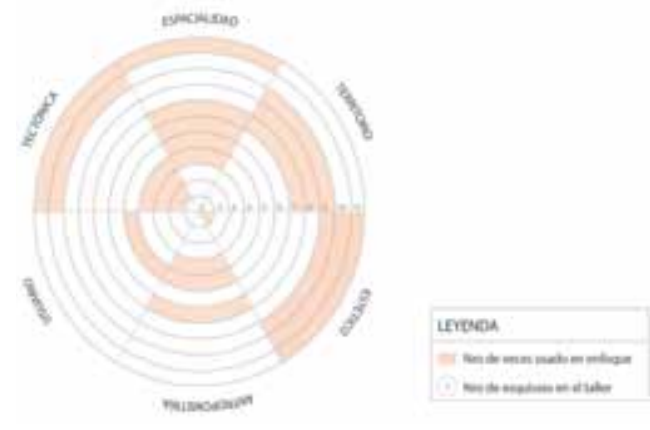

Figura 4. Enfoques de los esquisse en el taller de diseño 1 de la FAUA-UNI (2016-2) 
enfoques espacial y tectónico (ver figura 4). A su vez, aunque menos protagónica, se brinda de forma implícita el enfoque estético. Otros enfoques abarcados, aunque con menor intensidad, han sido el antropométrico y el territorial. Finalmente, aspectos sobre el usuario y función no se han considerado durante el respectivo ciclo (2016-2).

Con respecto al aspecto tectónico, son abarcados en los esquisse dos, tres, cuatro, diez y once. El taller toma en bastante consideración este enfoque (innato en la arquitectura, a diferencia de otras disciplinas artísticas) como el punto de partida inicial de los esquisse. A su vez, se vuelve a considerar en las dos dinámicas finales (esquisse 10 y 11) a modo de síntesis junto con otros enfoques. Este énfasis no es gratuito, pues su evaluación implica comprender la arquitectura como un objeto real que tendrá una posterior construcción. Por lo tanto, la arquitectura se fundamenta a través de sus lógicas constructivas y la naturaleza del material a utilizar.

Con respecto al enfoque espacial, se abarca desde el esquisse cuatro, cinco, seis, siete y once. El taller toma bastante consideración con este enfoque como la continuación del enfoque tectónico. A su vez, se vuelve a considerar en la última dinámica (esquisse 11) a modo de síntesis junto con el enfoque tectónico y estético. Este énfasis tiene como fin no solo la comprensión intuitiva del espacio al momento de proyectar las dinámicas, sino también la comprensión teórica, la cual es introducida al alumno desde el esquisse cuatro (4).

Finalmente, y como última etapa del taller, se abarca el enfoque territorial durante los esquisse siete (7), ocho (8) y nueve (9). En el esquisse 7, la presentación del territorio como variable resulta aún muy introductoria, pues el contexto inmediato aún no evidencia alguna relación directa (salvo la visual) con el recinto. Por otra parte, los esquisse 8 y 9 sí presentan al territorio como una variable que afecta directamente a la propuesta que el alumno va a generar o como protagonista de la dinámica. En esta etapa inicial del taller, se abarcó el concepto de territorio a partir de sus características físicas, así como por sus características perceptibles. Dentro de la primera, se reconocen estas variables físicas y mesurables como la topografía, el relieve y el asoleamiento. Por otro lado, entre las características perceptibles, el alumno considera, bajo su propio criterio, las visuales y el paisaje preferido a escoger.

En conclusión, cabe señalar que, en este nivel, el taller prefirió escoger entornos naturales, donde la intervención del hombre era nula o sin preexistencias de este. Esta condición permite obviar las características culturales que también existen en un territorio. Esta última caracteriza, si bien redefinen la imagen de lugar del alumno como un espacio inmaculado y ajeno al hombre, también complejiza en gran medida el concepto de territorio, el cual puede abarcarse con mayor énfasis en talleres posteriores.

\section{Taller de diseño 1-Laurín León Hilario - FAU- URP (2017-2)}

\section{Instrucciones}

Con respecto a las instrucciones en el Taller 1 del arquitecto Laurín León, cada ejercicio consiste en una duración de por lo menos 2 semanas. Esto se debe al acotado número de ejercicios que se realizan a lo largo del taller. Sin embargo, si bien la primera aproximación es "intuitiva", como lo describen los alumnos, esta se retroalimenta con las siguientes indicaciones que van señalando cada crítica hasta llegar al producto final. Finalmente, si bien no se ha realizado una encuesta sobre la imagen del taller por parte de los alumnos, muchos no consideraron favorable el nivel de abstracción que podía surgir a partir de las indicaciones. Esto se debe a que, desde la indicación, el alumno se confundía sobre cómo debía ser su producción o propuesta, y no podía reconocer las cualidades que le puedan señalar que su propuesta estaba bien o mal resuelta. Por otro lado, no se realizaba introducción teórica alguna al momento de iniciar algún aspecto fundamental en arquitectura por me- 
dio de los ejercicios. Por otra parte, tampoco se indicaban criterios para que el alumno pueda justificar sus intenciones estéticas formales. Estas, a su vez, se realizaban mediante dinámicas de trabajo grupal y colectivo.

\section{Formatos}
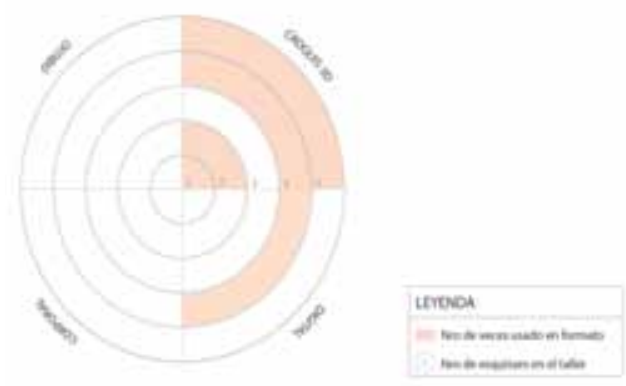

Figura 5. Formatos de los esquisse en el taller de diseño 1 de la FAU-URP (2017-1)

Con respecto a los formatos-tipologías, existe un marcado trabajo con el croquis tridimensional (ver figura 5). Sin embargo, tres de los cuatro croquis se realizan en una escala real, lo cual se relaciona con las intenciones del taller de generar experiencias en el alumno mediante la exploración y experimentación. Por otra parte, el esquisse cuatro es el único croquis que tiene una escala como condición para su realización, sin embargo, esta tenía la función de explorar de las dimensiones y espacios que se generarían posteriormente en el Habitáculo.

A su vez, para el esquisse tres (3), el taller opta por un formato digital, a través del software de diseño Rhinoceros. Si bien se comprende la importancia de los enfoques digitales en la formación del arquitecto, su introducción en un nivel de formación básico resulta dificil de comprender para los alumnos. Esto se explica, pues el alumno no está acostumbrado al software. Y, principalmente, el alumno no tiene noción sobre geometría descriptiva, el cual es un aspecto esencial para la comprensión no solo de los formatos digitales para arquitectura, sino además para el mismo dibujo proyectual (elevaciones, cortes, plantas, etc.). Por lo tanto, estos procesos se prefieren explorar a partir del formato gráfico del dibujo, pues resulta mucho más veloz y familiar para el alumno. Finalmente, el dibujo se abarca en el taller a partir de los bocetos realizados por el alumno en la bitácora.

\section{Funciones}

Los esquisse en el Taller de Diseño 1 de la FAU-URP (durante el ciclo 2017-1) consiste en un escaso número de ejercicios de larga duración (4 ejercicios). Por otra parte, no existen especificaciones por parte del taller sobre las temáticas vistas, tampoco hay un syllabus que lo indique; sin embargo, se reconocen los enfoques antropométricos bastante involucrados a lo largo del desarrollo de cada ejercicio. A su vez, la continuidad se reconoce por los aspectos estéticos formales presentes en cada ejercicio del taller. Finalmente, la función de cada ejercicio (y el taller) se puede describir como continuo, pues es bastante directa la hilación entre cada uno. Si bien en el esquisse tres (3), el formato varía a uno digital, esto no pausa la continuidad porque su resolución nace desde el proceso anterior. Por lo tanto, el ejercicio final (esquisse síntesis) resulta una consecuencia del proceso de aprendizaje del alumno mediante estos ejercicios.

\section{Enfoques}

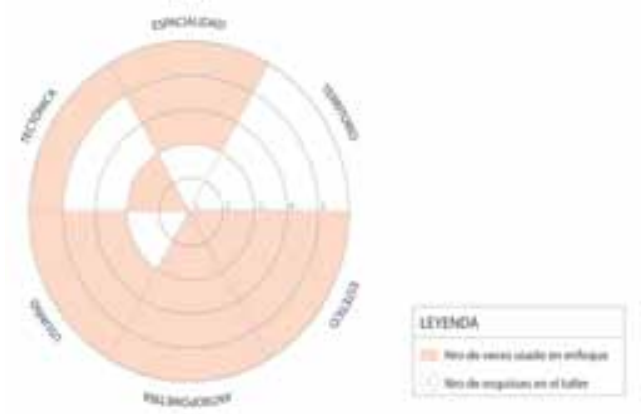

Figura 6. Enfoques de los esquisse en el taller de diseño 1 de la FAU-URP (2017-1) 
En el taller se realiza un menor número de ejercicios (5 ejercicios). Cada uno abarca más de tres enfoques, por lo que cada uno tiene un nivel de complejidad elevado. Sin embargo, se reconocen enfoques repetidos a lo largo de los esquisse como el estético-formal y el antropométrico (ver figura 6). Posteriormente, los enfoques espaciales, técnico-tectónico y usuario-función también se incorporan conforme se van añadiendo los ejercicios.

El enfoque antropométrico se abarca de forma continua a lo largo de todos lo ejercicio. Este da inicio en los esquisses uno y dos mediante el reconocimiento de los componentes del cuerpo humano para, finalmente, reproducirlo en un modelo que replique estos elementos. Por lo tanto, se trabaja en la noción del alumno sobre su propio cuerpo, a partir del reconocimiento de sus propias dimensiones y proporciones. A su vez, estos ejercicios exploran en aspectos más específicos como las articulaciones, los músculos y su estructura. Finalmente, resulta una aproximación más íntima, de la mano con la realización del croquis tridimensional en escala real.

Con respecto al enfoque estético-formal, durante el esquisse tres resulta complicado enfrentar las características estéticas, pues corresponde a un plano virtual de difícil exploración sin tener noción previa sobre geometría descriptiva. Sin embargo, independiente a este ejercicio, la exploración con los croquis tridimensionales permite una fácil comunicación entre la cátedra y los alumnos. Por otro lado, y gracias a los enfoques antropométricos abarcados de la mano, se puede reconocer una buena o mala ejecución del alumno gracias a la consideración del hombre como punto de medida (enfoque antropométrico).

El enfoque técnico-tectónico se identifica en el primer, segundo y quinto esquisse. Sin embargo, a pesar de estar en escala real, no se explota adecuadamente en los primeros dos ejercicios. Esto se debe a que no se trabaja a partir de la estructura del material, debido a que se enfocaba en los aspectos compositivos y antropométricos de este. El quinto ejercicio, sin embargo, sí explota el enfoque, pues el alumno aprende a partir de la experiencia de construir, considerando la madera como material básico. Por lo tanto, no solo se explota las propiedades del material, sino que el sistema constructivo propuesto debe poder funcionar como soporte estructural de una actividad a realizarse sobre este.

Finalmente, el enfoque espacial se explora desde el tercer esquisse, incorporándose a partir del desplazamiento del hombre. Sin embargo, el tercer esquisse se abarca el espacio virtual, y no el espacio físico. Por lo tanto, este termina siendo un ejercicio más compositivo. Por otro lado, el cuarto esquisse es un avance en cuanto ahora considera aspectos sobre la forma, las dimensiones y los límites formales. Sin embargo, la exploración más completa e interesante termina siendo el quinto esquisse, debido no solamente a que abarca todas las características propias de un enfoque espacial, sino que las evalúa mediante la construcción de un objeto en escala real. Si bien el formato real no es tan flexible como lo sería un croquis, este permite experimentar las sensaciones que genera un espacio real.

\section{Reflexiones}

A partir del análisis de los esquisses de cada taller, y su clasificación a partir de diferentes tipologías (tanto de función, formatos y enfoques), es posible perfilar las propuestas de enseñanza en arquitectura que cada una propone (ver tabla 1). De esta forma, es posible realizar una comparación objetiva sobre cómo los talleres afrontan los temas de enseñanza. Finalmente, si bien cada taller tiene una propuesta durante un determinado ciclo académico, estas estan en constante debate y replanteo por la misma cátedra.

Con respecto a los formatos, la propuesta del taller de diseño uno (1) de Aldo Mantovani Busato explora con la incorporación de un formato nuevo (corporal) que sería utilizado durante los primeros ejercicios antropométricos. Por lo tanto, se plantea una dualidad entre el formato y el enfoque abarcado, permitiendo 
Tabla 1. Formatos y enfoques de los esquisses de cada taller de diseño

\begin{tabular}{|c|c|c|c|c|c|c|c|c|c|c|c|c|}
\hline \multicolumn{13}{|c|}{ TALLER DE DISEÑO 1 FAUA-UNI (Manuel Flores Caballero) } \\
\hline \multicolumn{13}{|c|}{ ENFOQUES } \\
\hline & 1:dibujo & 2:columna & 3:puente & 4:refug & 5:cubo & 6:referente & 7:reflexión & 8:territorio & 9:recorrido & 10:superficie & 11: super + esp & TOTALX \\
\hline Tecnico tectónico & $=$ & $\frac{x}{x}$ & $\frac{x}{x}$ & $\frac{x}{x}$ & $=$ & $=$ & $=$ & $=$ & $=$ & $\frac{x}{x}$ & $\frac{x}{x}$ & 5 \\
\hline $\begin{array}{l}\text { Espacial } \\
\end{array}$ & " & "'-" & "'=" & $x$ & nex & $x$ & $x$ & "ume & "n+" & (") & na & 5 \\
\hline Antropométrico & $=$ & $=$ & $=$ & $x$ & $x$ & $=$ & $x$ & $=$ & $=$ & $=$ & $=$ & 3 \\
\hline Territorio & $=$ & $=$ & $=$ & $=$ & $=$ & $=$ & $x$ & $x$ & $x$ & $=$ & $=$ & 3 \\
\hline Estético formal & nats & "'t" & " "=" & "n+" & "s+" & " " & "'=" & "'=" & nats & taxt & 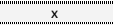 & 4 \\
\hline Usuario función & $=$ & $=$ & - & $=$ & $\mathrm{x}$ & $=$ & $=$ & $=$ & $=$ & - & $\mathrm{x}$ & 2 \\
\hline \multicolumn{13}{|c|}{ FORMATOS } \\
\hline & dibujo & 1:columna & 2:puente & 3:refug & 4:cubo & 5:referente & 6:reflexión & 7:territorio & 8:recorrido & 9:superficie & 10: super + esp & TOTALX \\
\hline Dibujo & $x$ & $=$ & $=$ & - & $=$ & $=$ & $=$ & $=$ & $=$ & $=$ & $=$ & 1 \\
\hline Croquis 3D & - & $x$ & $x$ & $x$ & $x$ & $x$ & $x$ & $x$ & $x$ & $x$ & $x$ & 10 \\
\hline Corporal & $=$ & $=$ & $=$ & $=$ & $=$ & $=$ & $=$ & $=$ & $=$ & $=$ & $=$ & 0 \\
\hline Digital & $=$ & $=$ & $=$ & $=$ & $=$ & $=$ & $=$ & $\mathrm{x}$ & $=$ & $=$ & $=$ & 1 \\
\hline \multicolumn{13}{|c|}{ TALLER DE DISEÑO 1 FAU-PUCP (Aldo Mantovani Busato) } \\
\hline \multicolumn{13}{|c|}{ ENFOQUES } \\
\hline & 1: piso & 2:hombre & 3. equilibrio & 4: aparato & 5: hom-arbol & 6: bosque & 7: cajas & - & - & - & - & TOTALX \\
\hline Tecnico tectónico & $=$ & $=$ & $=$ & $=$ & $=$ & $=$ & $=$ & $=$ & $=$ & $=$ & $=$ & 0 \\
\hline Espacial & - & 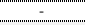 & $\because=$ & $\because=$ & $\because=$ & $\because=$ & $x$ & $y=$ & - & 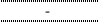 & - & 1 \\
\hline Antropométrico & $x$ & $x$ & $x$ & $x$ & $x$ & $x$ & $x$ & $=$ & $=$ & $=$ & $=$ & 7 \\
\hline Territorio & $=$ & $=$ & $=$ & $=$ & $x$ & $x$ & $=$ & $=$ & $=$ & $=$ & $=$ & 2 \\
\hline Estético formal & "'=" & " & " =" & $=$ & "'-" & " =" & $x$ & "=" & "n+" & $=$ & " & 1 \\
\hline Usuario función & - & $=$ & $=$ & $=$ & $\mathrm{x}$ & $\mathrm{x}$ & $\mathrm{x}$ & $=$ & $=$ & $=$ & $=$ & 3 \\
\hline \multicolumn{13}{|c|}{ FORMATOS } \\
\hline & 1: piso & 2:hombre & 3. equilibrio & 4: aparato & 5: hom-arbol & 6: bosque & 7: cajas & - & - & - & - & TOTALX \\
\hline Dibujo & $=$ & $\frac{x}{x}$ & $\frac{x}{x}$ & $x$ & $\frac{x}{x}$ & $\frac{x}{x}$ & $x$ & $=$ & $=$ & $=$ & $=$ & 6 \\
\hline Croquis 3D & - & - & $\because=$ & $x$ & $x$ & $x$ & $x$ & $=$ & $y=$ & $y=$ & $1=$ & 4 \\
\hline Corporal & $x$ & $=$ & $x$ & $=$ & $=$ & $=$ & $=$ & $=$ & $=$ & $=$ & $=$ & 2 \\
\hline Digital & $=$ & $=$ & $=$ & - & $=$ & $=$ & $=$ & $=$ & $=$ & $=$ & $=$ & 0 \\
\hline \multicolumn{13}{|c|}{ TALLER DE DISEÑO 1 FAU-URP (Laurín León Hilario) } \\
\hline \multicolumn{13}{|c|}{ ENFOQUES } \\
\hline & 1: la mano & 2: clon & 3:esp virtual & 4:maqueta & $5: 1 / 1$ & - & - & - & - & - & - & TOTALX \\
\hline Tecnico tectónico & $x$ & $x$ & $=$ & $=$ & $x$ & $=$ & $=$ & $=$ & $=$ & $=$ & - & 3 \\
\hline Espacial & $=$ & $=$ & $x$ & $x$ & $x$ & $=$ & $=$ & $=$ & $=$ & $=$ & $=$ & 3 \\
\hline Antropométrico & $x$ & $x$ & $x$ & $x$ & $x$ & $=$ & $=$ & $=$ & $=$ & $=$ & $=$ & 5 \\
\hline Territorio & (n) & (nt) & (n) & (n) & (n) & 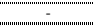 & "'=" & = & $=$ & $=$ & $=$ & 0 \\
\hline Estético formal & $x$ & $x$ & $x$ & $x$ & $x$ & $=$ & $=$ & $=$ & $=$ & $=$ & $=$ & 5 \\
\hline Usuario función & - & - & $x$ & $x$ & $x$ & - & $=$ & $=$ & $=$ & $=$ & $=$ & 3 \\
\hline \multicolumn{13}{|c|}{ FORMATOS } \\
\hline & 1: la mano & 2: clon & 3:esp virtual & 4:maqueta & $5: 1 / 1$ & - & - & - & $\overline{-}$ & $=$ & - & TOTALX \\
\hline Dibujo & $=$ & $=$ & $=$ & $=$ & $=$ & $=$ & $=$ & $=$ & $=$ & $=$ & $=$ & 0 \\
\hline Croquis 3D & $x$ & $x$ & $=$ & $x$ & $x$ & 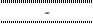 & name & 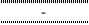 & matum & $=$ & $=$ & 4 \\
\hline Corporal & $=$ & $=$ & $=$ & $=$ & $=$ & $=$ & $=$ & $=$ & $=$ & $=$ & $=$ & 0 \\
\hline Digital & $=$ & $=$ & $x$ & $=$ & $=$ & $=$ & - & $=$ & $=$ & $=$ & $=$ & 1 \\
\hline
\end{tabular}

Elaboración propia

al alumno ser conciente de las dimensiones y proporciones de su cuerpo, tomándolo directamente como objeto de la dinámica. Por otro lado, los formatos de la mayoría de esquisses propuestos tenían al dibujo como la herramienta adjunta indispensable. Esta dualidad entre formatos, permitía acercar al alumno al lenguaje para exponer sus intenciones.

Con respecto a los enfoques, si bien aspectos como la antropometría, el territorio y la tectónica, son considerados por encima del resto de enfoques, todos los talleres han culminado con uno o más ejercicios de enfoque espacial. Esto responde al espacio como característica intrínseca en la enseñanza en arquitectura. Por lo tanto, su presencia en el taller de diseño es considerada obligatoria.
Por otro lado, el taller de Laurín León y el de Manuel Flores tienen propuestas diferentes sobre los enfoques abordados por cada esquisse. Esto se debe a que el taller de diseño 1 de Manuel plantea los enfoques a modo de temáticas, permitiendo al alumno concentrarse en cada enfoque a lo largo de ejercicios específicos hasta pasar al nuevo tema. Por el contrario, el taller de diseño 1 de Laurín León, propone un pequeño número de ejercicios, en cuya solución, aborde varios enfoques. Sin embargo, el taller de diseño 1 de Aldo Mantovani, va abordando cada concepto en arquitectura a partir de la hilación con el enfoque antropométrico, el cual está presente a lo largo de cada esquisse.

Finalmente, el esquisse síntesis se ve presente en los tres talleres de diseño (aunque en 
el taller de Manuel Flores se aborda como un pequeño proyecto arquitectónico). Es así que estos cumplen la función de sintetizar los temas abordados por cada taller en la elaboración de una dinámica final. Este último ejercicio se debe incluir de forma obligatoria todos los contenidos del taller.

\section{Referencias}

Bertero, C. (2012). La enseñanza de la arquitectura: entre lo dibujado y lo desdibujado. Santa Fe, Argentina: Universidad Nacional del Litoral.

Beynon, D., \& Tucker, R. (2012). The Esquisse. In Assessing creativity: supporting learning in architecture and design (pp. 177-185). Office for Learning and Teaching. Recuperado de https:// www.researchgate.net/publication/305058995_ Esquisse

Chafee, R. (1977). La esperanza de la arquitectura en L'école Des Beaux-arts. In La arquitectura de la Ecole des Beaux-Arts. New York: Museum of Modern Art. Recuperado de https://es.scribd. $\mathrm{com} / \mathrm{doc} / 139619356 / \mathrm{la}-\mathrm{ensenanza-de-la-}$ arquitectura-en-la-ecole-des-beaux-arts?doc id=139619356\&order $=470752596$

Ching, F. (2006). Arquitectura: Forma, espacio y orden. 2 da ed. Barcelona, España: Gustavo Gili.
Dreifuss, C. (2011). Aspectos estéticos en la enseñánza del primer taller de diseño. Investigaciones Ciudad \& Arquitectura. 1, 63-67.

Dreifuss, C. (2015). Enseñanza-aprendizaje en el taller de diseño. Limaq, 1, 67-92. Recuperado de https://revistas.ulima.edu.pe/index.php/Limaq/ article/viewFile/354/337

Fabbri, M., \& Tapia, K. (2015). Los talleres básicos de la Universidad de Lima: algunas reflexiones en torno a los procesos de enseñanzaaprendisaje. Limaq. 1, 111-125.

Muñoz, A. (2008). El proyecto de arquitectura: concepto, proceso y representación. Barcelona, España: Editorial Reverté.

Ramon, A. (2002). Función. En Introduccion a la arquitectura: Conceptos fundamentales (pp. 109-126). Mexico: Alfaomega. 